(1)

CrossMark

\title{
Avoiding immortal time bias in observational studies
}

\author{
To the Editor:
}

We read with interest the recent study by SuISSA et al. [1] published in the European Respiratory Journal. The study used population-based administrative data from the province of Quebec, Canada, to evaluate the association between inhaled corticosteroid (ICS) exposure and lung cancer risk in patients with COPD. The results of the study indicated that ICS use was not associated with lung cancer risk in this patient population.

Our particular interest in reading this study stems from our own work published in the ERJ, which addressed the same question using administrative health data from the province of British Columbia, Canada, but showed different results [2]. A major strength of our study was that we directly linked health administrative data with the British Columbia Cancer Registry file, which enabled us to accurately ascertain the date of the lung cancer diagnosis, as well as the histological subtype of tumour for each patient (discussed further below), and employed multiple methods of measuring exposure. Our results indicate a protective effect resulting from ICS exposure on lung cancer risk across several time-dependent methods of defining medication exposure.

Suissa et al. [1] draw attention to the concept of immortal time bias in our analysis as a possible explanation for the discordant findings between their results and ours. The authors have compiled a commendable body of research identifying immortal time bias in observational studies, particularly in studies where cancer is an outcome. Immortal time bias arises in different contexts, but commonly when the definition of exposure is satisfied at some point during the follow-up time but is applied to the entire follow-up period, thus requiring patients to survive (and overestimating time considered as exposed) until the time of exposure (additional details and discussion of immortal time bias available in $[3,4]$ ). This is, unfortunately, an all too common occurrence in observational studies, particularly those using administrative data.

However, we would like to objectively refute the claim that our design suffered from immortal time bias. The authors cite the following phrase from our study: “...based on having filled a prescription after the start of follow-up”, failing to cite the previous sentence which clearly states: “The time-dependent 'ever' exposure..." explicitly stating that our exposure definition was time-dependent. Thus, our analysis was not based on a "fixed" exposure definition, but rather ICS exposure was defined as a time-dependent variable. This point was explicitly stated throughout our report (see the "Abstract", "Methods" section entitled "ICS exposure", table 3, table 4, table 5 and table 6). To enable others to view our approach, we have also posted the relevant section of our analysis code online (https://sites.google.com/view/analysiscode-raymakersetal2019/ home). Our approach appropriately classifies an individual as unexposed during the time after entry into the cohort and before the first ICS prescription, which avoids immortal time bias.

The question remains as to why the study by SuIssa et al. [1] arrived at a different conclusion from our own. One possible difference is that our identification of lung cancer cases was obtained from a directly linked cancer registry database and as such, our lung cancer diagnoses were histopathologically confirmed, whereas lung cancer diagnoses in SuISSA et al. [1] were on the basis of hospital discharges. If the diagnosis of lung cancer using these records might include additional diagnoses beyond lung cancer, specifically diagnoses that would not be affected by ICS exposure, there could be an ascertainment bias towards a null effect.

In closing, we certainly appreciate the expertise of the authors and their previous contributions to this area of research; however, we feel that in this instance they have misunderstood important details of our analysis.

@ERSpublications

Immortal time bias is common in observational studies; however, in the analysis conducted by Raymakers and colleagues appropriate measures were taken to avoid this bias http://bit.ly/36042OV

Cite this article as: Raymakers AJN, Sadatsafavi M, Sin DD, et al. Avoiding immortal time bias in observational studies. Eur Respir J 2020; 55: 1902494 [https://doi.org/10.1183/13993003.02494-2019]. 
As such, we feel the need to draw attention to this misunderstanding and state explicitly to the readers of the ERJ that our analysis used appropriate methods to avoid immortal time bias.

\author{
Adam J.N. Raymakers ${ }^{1}$, Mohsen Sadatsafavi ${ }^{1}$, Don D. $\operatorname{Sin}^{2,3}$, J. Mark FitzGerald ${ }^{3}$, Carlo A. Marra ${ }^{4}$ and \\ Larry D. Lynd ${ }^{1,5}$ \\ ${ }^{1}$ Collaboration for Outcomes Research and Evaluation (CORE), Faculty of Pharmaceutical Sciences, University of British \\ Columbia, Vancouver, BC, Canada. ${ }^{2}$ Centre for Heart Lung Innovation, St Paul's Hospital, Vancouver, BC, Canada. \\ ${ }^{3}$ Division of Respiratory Medicine, Faculty of Medicine, University of British Columbia, Vancouver, BC, Canada. \\ ${ }^{4}$ School of Pharmacy, University of Otago, Dunedin, New Zealand. ${ }^{5}$ Centre for Health Evaluation and Outcome \\ Sciences, Providence Health Research Institute, Vancouver, BC, Canada.
}

Correspondence: Adam J.N. Raymakers, Collaboration for Outcomes Research and Evaluation (CORE), Faculty of Pharmaceutical Sciences, University of British Columbia (UBC), 2405 Wesbrook Mall, Vancouver, BC, Canada, V6T1Z3. E-mail: araymakers@bccrc.ca

Received: 27 Dec 2019 | Accepted: 06 Jan 2020

Conflict of interest: A.J.N. Raymakers has nothing to disclose. M. Sadatsafavi reports grants and personal fees from GlaxoSmithKline and AstraZeneca, outside the submitted work. D.D. Sin reports grants from Merck, personal fees for advisory board work from Sanofi-Aventis and Regeneron, grants and personal fees for lectures and advisory board work from Boehringer Ingelheim and AstraZeneca, personal fees for lectures and advisory board work from from Novartis, outside the submitted work. J.M. FitzGerald has nothing to disclose. C.A. Marra has nothing to disclose. L.D. Lynd reports grants from Canadian Institute for Health Research, during the conduct of the study; grants from AstraZeneca, outside the submitted work.

\title{
References
}

1 Suissa S, Dell'Aniello S, Gonzalez AV, et al. Inhaled corticosteroid use and the incidence of lung cancer in COPD. Eur Respir J 2020; 55: 1901720.

2 Raymakers AJN, Sadatsafavi M, Sin DD, et al. Inhaled corticosteroids and the risk of lung cancer in COPD: a population-based cohort study. Eur Respir J 2019; 53: 1801257.

3 Lévesque LE, Hanley JA, Kezouh A, et al. Problem of immortal time bias in cohort studies: example using statins for preventing progression of diabetes. BMJ 2010; 340: b5087.

4 Rothman KJ, Greenland S, Lash TL. Modern Epidemiology. 3rd Edn. Philadelphia, Lippincott Williams \& Wilkins, 2008.

Copyright @eERS 2020

\section{From the authors:}

We thank A.J.N. Raymakers and co-workers for their letter, which clarifies the method of data analysis used in their study of the association between inhaled corticosteroids (ICS) and lung cancer incidence in COPD [1]. Indeed, as shown by the computing code they provide, the data analysis did consider ICS use as a time-dependent exposure, thus avoiding immortal time bias.

Our assumption that this bias affected their study arose mainly because of the scarcity in data presentation, in particular the crude rates. The Strengthening the Reporting of Observational Studies in Epidemiology (STROBE) guidelines make extensive recommendations that allow to better understand and interpret data from observational studies, thus avoiding such misunderstandings [2]. In particular, it advocates that in "a cohort study with events as outcomes, report the numbers of events for each outcome of interest ... the event rate per person-year of follow-up ... presenting such information separately for participants in different exposure categories of interest ... giving the unadjusted analyses together with the main data" [2]. A presentation of these data by A.J.N. Raymakers and co-workers, including information on the person-time and crude rates before and after ICS initiation, would have mitigated concerns about potential immortal time bias in the design and analysis of this observational study.

We agree that the availability of a cancer registry, such as the excellent one from British Columbia, with the confirmed diagnoses used in their study, provides more accurate outcome events than claims-based

@ERSpublications

Raymakers and colleagues used the proper analysis that avoided immortal time bias, though the wide discrepancy in results with other studies remains; further studies are needed http://bit.ly/2Oobgqb

Cite this article as: Suissa S, Ernst P. Avoiding immortal time bias in observational studies. Eur Respir J 2020; 55: 2000138 [https://doi.org/10.1183/13993003.00138-2020]. 
diagnoses. Nevertheless, validation studies in other databases have shown good accuracy for the diagnosis of lung cancer from claims data [3,4]. This suggests that the resulting magnitude of the potential dilution of the hazard ratio towards the null in our study is likely marginal.

In all, it is reassuring to find out that the study of RAYMAKERS et al. [1] used the proper time-dependent analysis that avoided immortal time bias. On the other hand, the relatively good accuracy of the diagnosis using claims data in our study likely does not fully explain the wide discrepancy in results between the two studies, namely a 30\% reduction versus no reduction in lung cancer incidence with ICS use [5]. Thus, in view of our and other studies that also found no reduction in lung cancer incidence with ICS use, further research is needed to better understand this association, particularly if the suggestion of a randomised trial is contemplated [6].

Samy Suissa ${ }^{1,2}$ and Pierre Ernst ${ }^{1,2}$

${ }^{1}$ Center for Clinical Epidemiology, Lady Davis Institute - Jewish General Hospital, Montreal, QC, Canada. ${ }^{2}$ Dept of Epidemiology and Biostatistics and Dept of Medicine, McGill University, Montreal, QC, Canada.

Correspondence: Samy Suissa, Centre for Clinical Epidemiology, Jewish General Hospital, 3755 Cote Ste-Catherine, Montreal, Québec, Canada H3T 1E2. E-mail: samy.suissa@mcgill.ca

Received: 22 Jan 2020 | Accepted: 22 Jan 2020

Conflict of interest: S. Suissa reports grants and personal fees for advisory board work and lectures from Boehringer Ingelheim and Novartis, personal fees for lectures from AstraZeneca, outside the submitted work. P. Ernst has nothing to disclose.

\section{References}

1 Raymakers AJN, Sadatsafavi M, Sin DD, et al. Inhaled corticosteroids and the risk of lung cancer in COPD: a population-based cohort study. Eur Respir J 2019; 53: 1801257.

2 Vandenbroucke JP, von Elm E, Altman DG, et al. Strengthening the Reporting of Observational Studies in Epidemiology (STROBE): Explanation and Elaboration. Epidemiology 2007; 18: 805-835.

3 Setoguchi S, Solomon DH, Glynn RJ, et al. Agreement of diagnosis and its date for hematologic malignancies and solid tumors between medicare claims and cancer registry data. Cancer Causes Control 2007; 18: 561-569.

4 Montedori A, Bidoli E, Serraino D, et al. Accuracy of lung cancer ICD-9-CM codes in Umbria, Napoli 3 Sud and Friuli Venezia Giulia administrative healthcare databases: a diagnostic accuracy study. BMJ Open 2018; 8: e020628.

5 Suissa S, Dell'Aniello S, Gonzalez AV, et al. Inhaled corticosteroid use and the incidence of lung cancer in COPD. Eur Respir J 2020; 55: 1901720.

6 Seijo LM, Soriano JB, Peces-Barba G. New evidence on the chemoprevention of inhaled steroids and the risk of lung cancer in COPD. Eur Respir J 2019; 53: 1900717. 